\title{
Nonradiative Energy Transfer between Doped and Undoped Flat Semiconductor Nanocrystals of Colloidal Quasi-2D Nanoplatelets
}

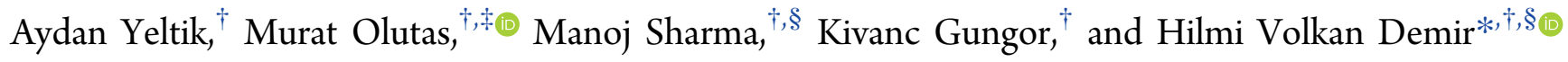 \\ ${ }^{\dagger}$ Department of Physics, Department of Electrical and Electronics Engineering, and UNAM-Institute of Materials Science and \\ Nanotechnology, Bilkent University, Ankara 06800, Turkey \\ ${ }^{\ddagger}$ Department of Physics, Bolu Abant Izzet Baysal University, Bolu 14030, Turkey \\ ${ }^{\S}$ LUMINOUS! Center of Excellence for Semiconductor Lighting and Displays, School of Electrical and Electronics Engineering, \\ School of Physical and Mathematical Sciences, Nanyang Technological University, Nanyang Avenue, 639798, Singapore
}

Supporting Information

ABSTRACT: Atomically flat colloidal semiconductors such as nanoplatelets (NPLs) promise great potential for different optoelectronic applications. Here, we systematically investigate the excitonic energy transfer from colloidal $\mathrm{Cu}$-doped $\mathrm{CdSe}$ to undoped core/shell $\mathrm{CdSe} / \mathrm{CdS}$ nanoplatelets via steady-state and time-resolved photoluminescence spectroscopy techniques. We show the strong quenching in photoluminescence emission of the doped NPL donors together with significant modifications in the time-resolved kinetics by changing the concentration of the undoped NPL acceptors in close proximity. This newly presented all-colloidal and all-quasi2D doped-undoped NPL-NPL hybrid system shows near-unity room-temperature energy transfer efficiency ( $99 \%$ ) in solid films. We strongly believe that such highly efficient energy transfer in doped-undoped hybrid films will create more interest in the scientific community to further explore different donor/acceptor combinations with these newly reported doped NPLs for next-generation energy harvesting applications.

\section{INTRODUCTION}

Colloidal luminescent quasi-two-dimensional (quasi-2D) semiconductor quantum wells, which are commonly known as nanoplatelets (NPLs), have recently attracted great attention as a new class of semiconductor nanocrystals (NCs). ${ }^{1-12}$ Besides, the extensive investigation of group II-VI undoped and doped zero-dimensional NCs, ${ }^{13-20}$ CdSe NPLs with zincblende structure have also been studied in terms of optical and excitonic properties, particularly important for optoelectronic applications. ${ }^{1,2,8-12,21}$ These core-only NPLs have superior features including spectrally narrow emission ( $\mathrm{fwhm}<10 \mathrm{~nm}$ ) because of the precise thickness control in 1D, large absorption cross-section, giant oscillator strength, and high packing factor. ${ }^{1,2,11,22}$ Furthermore, core/shell CdSe/CdS NPLs possess several other important properties such as high surface passivation resulting in high quantum yield, and reduced emission intermittency at a single nanoparticle level. ${ }^{7}$ Therefore, core-only CdSe and core/shell CdSe/CdS NPLs offer strong potential as favorable candidates in various applications including light emitting diodes, solar cells, low-threshold

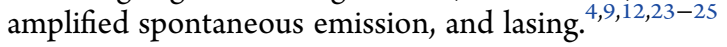

Efficient ion doping of core-only CdSe NPLs has been demonstrated in very recent reports by Sharma et al. through the inclusion of copper $(\mathrm{Cu})$ ions in the synthesis. ${ }^{26,27} \mathrm{Cu}$-doped CdSe NPLs provide significant characteristics such as large Stokes-shifted and tunable emission spectrum in the range of visible to near infrared, near-unity quantum efficiency, and step-
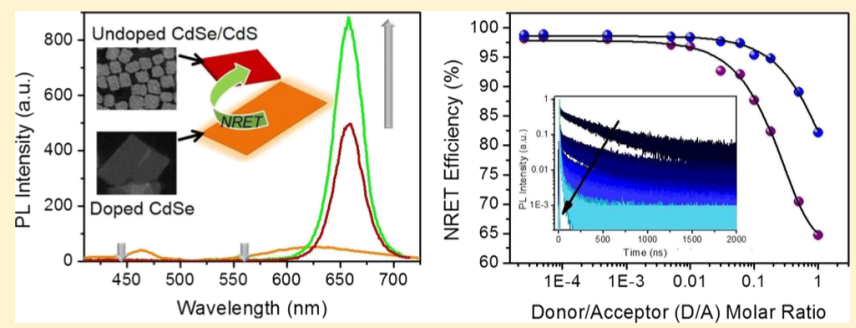

Donor/Acceptor (D/A) Molar Ratio like absorption feature. ${ }^{26}$ As expected from doped semiconductor NCs, Cu-related emission in the doped NPLs is much broader than the band-edge emission. ${ }^{27-29}$ Doped NPLs might also have an order of magnitude longer excited state lifetime and much lower self-absorption when compared to undoped NPLs. ${ }^{7,26}$ Considering the promising features of the doped and undoped NPLs, highly enhanced Förster-type nonradiative energy transfer (NRET) is to be observed in the heterostructures of $\mathrm{Cu}$-doped core-only $\mathrm{CdSe} \mathrm{NPLs}$ as the exciton donors and undoped core-shell CdSe/CdS NPLs as the exciton acceptors, which is particularly important for nextgeneration light harvesting applications. In addition, $\mathrm{Cu}$-doped cadmium-based NPLs may provide a facile platform for the investigation of charge carrier dynamics in the pairs of colloidal quasi-2D doped-donor and undoped-acceptor through the nearfield interaction. ${ }^{30-32}$

Pradhan and co-workers previously investigated the fluorescence energy transfer between doped and undoped QDs, and the energy transfer efficiency was reported as around $39 \% .{ }^{33}$ However, the energy transfer mechanism in the doped/undoped NPL heterostructures, which may offer superior characteristics compared to the QD heterostructures, has not been studied to date. Furthermore, besides their stable and dominant dopant

Received: October 18, 2018

Revised: December 18, 2018

Published: December 21, 2018 


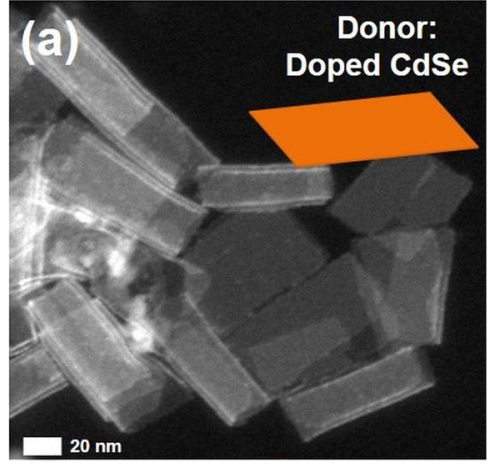

(c)

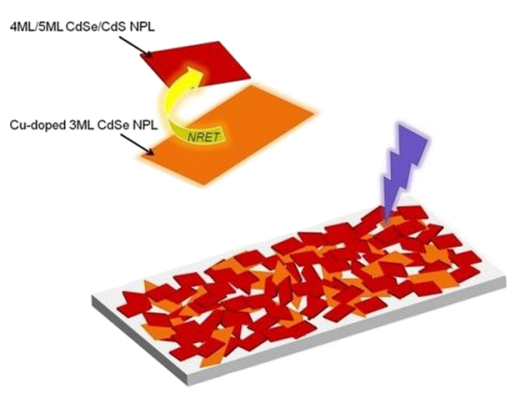

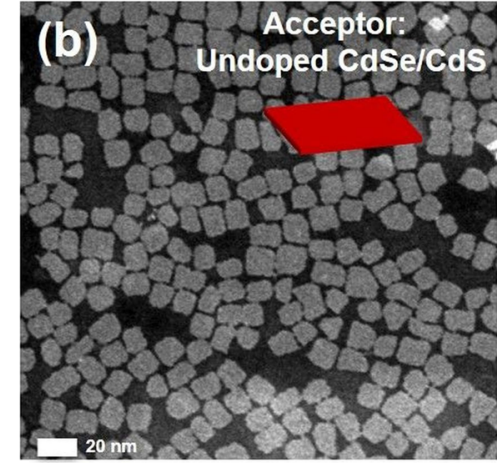

(d)

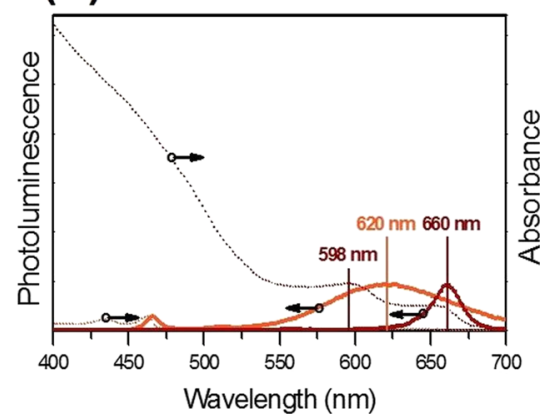

Figure 1. TEM images of the (a) Cu-doped 3 ML core NPLs and (b) undoped 4 ML/5 ML CdSe/CdS core/shell NPLs. (c) Schematic of our hybrid structure including the $\mathrm{Cu}$-doped and undoped NPLs. (d) PL and absorbance spectra of the Cu-doped and undoped NPLs dispersed in toluene. Here, the PL spectra of donor and acceptor NPLs together with the absorption spectrum of acceptor NPLs were normalized to each other using Cu-related emission peak value of the donor, band-edge emission peak value of the acceptor and second excitonic absorption peak value of the acceptor.

related emission, $\mathrm{Cu}$-doped NPLs having lower self-absorption because of the large Stokes-shifted emission and longer excited state lifetime when compared to the undoped donor NPLs have not been utilized for the efficient NRET in heterostructures. ${ }^{12,26}$ In this study, we investigated the NRET from colloidal $\mathrm{Cu}$ doped CdSe NPLs to undoped CdSe/CdS NPLs. The record high energy transfer efficiency, $\sim 99 \%$, is reported here in the doped/undoped NPL solid films via time-resolved fluorescence (TRF) spectroscopy conducted at room temperature. Strong NRET is also demonstrated via steady-state photoluminescence (PL) emission spectroscopy exhibiting the transfer efficiency above $90 \%$ for an optimized donor/acceptor (D/A) ratio. In addition, steady-state PL excitation spectroscopy was used to further confirm the efficient quenching of the PL from $\mathrm{Cu}$ related trap in the proposed solids.

\section{EXPERIMENTAL SECTION}

Chemicals. Cadmium nitrate tetrahydrate $\left(\mathrm{Cd}\left(\mathrm{NO}_{3}\right)_{2}\right.$. $\left.4 \mathrm{H}_{2} \mathrm{O}\right)(99.999 \%$ trace metals basis), sodium myristate (>99\%), technical-grade 1-octadecene (ODE), selenium ( $\mathrm{Se}$ ) (99.999\% trace metals basis), cadmium acetate dihydrate $\left(\mathrm{Cd}(\mathrm{OAc})_{2} \cdot 2 \mathrm{H}_{2} \mathrm{O}\right)(>98 \%)$, technical-grade oleic acid (OA) (90\%), technical-grade oleylamine (OAm) (70\%), N-methylformamide (NMF) (99\%), and ammonium sulfide solution (40-48 wt \% in $\mathrm{H}_{2} \mathrm{O}$ ), copper(II) acetate (99.999\%) were purchased from Sigma-Aldrich. Hexane, ethanol, methanol, toluene, and acetonitrile were purchased from Merck Millipore and used without any further purification.

Synthesis of Cu-Doped 3 ML CdSe NPLs. $0.5 \mathrm{mmol}$ of $\mathrm{Cd}(\mathrm{Ac})_{2} \cdot 2 \mathrm{H}_{2} \mathrm{O}, 0.3 \mathrm{mmol}$ of $\mathrm{OA}$ and $15 \mathrm{~mL}$ of ODE were mixed in a $50 \mathrm{~mL}$ three-neck flask. The mixture was stirred under continuous Ar bubbling for $10 \mathrm{~min}$. Afterward $0-200 \mu \mathrm{L}$ of $\mathrm{Cu}$ precursor was added to the reaction mixture at room temperature. Subsequently, under Ar protection, the flask was heated to $230{ }^{\circ} \mathrm{C}$ in $5 \mathrm{~min}$. Thereafter, the flask was kept at 230 ${ }^{\circ} \mathrm{C}$ for another $5 \mathrm{~min}$ and $2.5 \mathrm{~mL}$ of $3 \mathrm{mmol} \mathrm{Se}-\mathrm{ODE}$ solutions was injected. Light yellow color appears immediately after the injection of the Se precursor. Following the growth for $5 \mathrm{~min}$, the solution temperature was decreased to room temperature using a water bath. After adding $5 \mathrm{~mL}$ of toluene into the solution, it was centrifuged for $10 \mathrm{~min}$ at $2500 \mathrm{rpm}$ and the supernatant was removed into another centrifuge tube. The supernatant was removed into another centrifuge tube and ethanol was added into supernatant solution till it became turbid. Thereafter, this solution was centrifuged again at $8000 \mathrm{rpm}$ for $5 \mathrm{~min}$, and then the precipitates of 3 monolayer (ML) Cu doped CdSe NPLs were dissolved and stored in toluene for further use. The Se precursor used in the synthesis was prepared according to a previously reported procedure by the group of Peng in their work, Nano Lett. 15, 4477 (2015). ${ }^{34}$ For the Cu precursor utilized in the synthesis of the doped NPLs, $15 \mathrm{mg}$ of copper (II) acetate was first mixed with $0.2 \mathrm{~mL}$ of oleylamine and $5 \mathrm{~mL}$ ODE in an $\mathrm{N}_{2}$ filled glovebox environment. The mixture was then stirred at $90{ }^{\circ} \mathrm{C}$ for $24 \mathrm{~h}$.

Synthesis of $4 \mathrm{ML}$ CdSe NPLs. $340 \mathrm{mg}$ of cadmium myristate $\left(\mathrm{Cd}(\mathrm{myr})_{2}\right), 24 \mathrm{mg}$ of selenium $(\mathrm{Se})$ and $30 \mathrm{~mL}$ of octadecene (ODE) were introduced into a $100 \mathrm{~mL}$ three-neck flask, and the mixture was degassed under vacuum at $95{ }^{\circ} \mathrm{C}$ for an hour until it was completely dissolved. After degassing, the solution was heated to $240{ }^{\circ} \mathrm{C}$ under argon atmosphere, and 120 $\mathrm{mg}$ of cadmium acetate dehydrate $\left(\mathrm{Cd}(\mathrm{OAc})_{2}\left(\mathrm{H}_{2} \mathrm{O}\right)_{2}\right)$ was introduced swiftly into the hot mixture when the temperature reached $195{ }^{\circ} \mathrm{C}$. After $10 \mathrm{~min}$ of growth at $240^{\circ} \mathrm{C}, 1 \mathrm{~mL}$ of OA was added and then the mixture was quickly cooled down to room temperature. After adding $5 \mathrm{~mL}$ of hexane into the solution, it was centrifuged for $6 \mathrm{~min}$ at $6000 \mathrm{rpm}$. The 
supernatant was removed into another centrifuge tube and ethanol was added into the supernatant solution until it became turbid. The turbid solution was centrifuged at $10000 \mathrm{rpm}$ for 10 $\mathrm{min}$, and then the precipitate was dissolved in hexane and stored for the further $\mathrm{CdS}-$ shell growth.

Synthesis of $4 \mathrm{ML} / 5 \mathrm{ML} \mathrm{CdSe} / \mathrm{CdS}$ Core/Shell NPLs. $\mathrm{CdS}$-shell growing of the $4 \mathrm{ML}$ CdSe core-only NPLs were carried by using the colloidal atomic layer deposition technique. $^{35}$ First, for a typical CdS-shell deposition, $3 \mathrm{~mL}$ of 4 ML CdSe seed NPLs and $3 \mathrm{~mL}$ of NMF were mixed. Then, the NPLs were transferred from hexane to NMF solution with the addition of $50 \mu \mathrm{L}$ of ammonium sulfide solution $\left(\mathrm{S}\left(\mathrm{NH}_{4}\right)_{2}\right)$. After 5 min shaking and stirring for complete reaction, $500 \mu \mathrm{L}$ acetonitrile and $3 \mathrm{~mL}$ toluene were introduced into the mixture, and they were centrifuged for $5 \mathrm{~min}$ at $8000 \mathrm{rpm}$ to precipitate the NPLs. For complete removal of the excess sulfur precursor, the washing step was repeated three times. After the precipitated NPLs were dissolved in $3 \mathrm{~mL}$ of NMF, $2 \mathrm{~mL}$ of cadmium precursor (0.2 M cadmium acetate-NMF) was added and shaked for complete reaction. The NPLs were precipitated again with the addition of acetonitrile and toluene. After that, $1 \mathrm{ML}$ CdS-shell is formed on CdSe NPLs. For further increasing the $\mathrm{CdS}$-shell thickness, this process is followed in the same way. Finally, CdSe/CdS core/shell NPLs were precipitated and dispersed in toluene with the addition of excess amount of $\mathrm{OA}$.

TRF, Steady-State PL Emission/Excitation and Absorbance Spectroscopy. A time correlated single photon counting system having time resolution down to 4 ps (PicoHarp 300) and capable of delivering laser pulses with $80 \mathrm{MHz}$ repetition rate was used for the TRF spectroscopy measurements. The system includes a picosecond pulsed laser with an output photon energy of $3.31 \mathrm{eV}(375 \mathrm{~nm})$ driven by a driver module (PDL-800 series), and a fast photomultiplier tube (Hamamatsu H5783 series) to be able to resolve the lifetimes on the order of a few picoseconds. In the TRF spectroscopy measurements, the exciton density per NPL is very small $(\langle\mathrm{N}\rangle \ll 1)$ owing to the low intensity of the pump laser. Cary $100 \mathrm{UV}-\mathrm{Vis}$ spectrophotometer including a xenon lamp, a monochromator, and a standard photomultiplier tube was used to obtain the results of steady-state PL emission/excitation and UV-Vis absorbance spectroscopy. Excitation wavelength was fixed to $350 \mathrm{~nm}$ in the steady-state PL emission and UV-Vis absorbance spectroscopy measurements.

\section{RESULTS AND DISCUSSIONS}

The transmission electron microscopy (TEM) images of the Cudoped 3 ML CdSe core NPLs and undoped $4 \mathrm{ML} / 5 \mathrm{ML}$ CdSe/ $\mathrm{CdS}$ core/shell NPLs are shown in Figure 1a,b, respectively. As seen from the figures, any significant self-stacking was not observed in the solids of both the donor and acceptor NPLs except some donor NPLs touching to each other because of their relatively high aspect ratio in size. A schematic of our experimental system including the doped and undoped NPLs is depicted in Figure 1c. Ensemble of the Cu-doped NPLs with the absolute PL QE of 70\% has Cu-state-related PL at around $620 \mathrm{~nm}$ along with the band-edge emission at around $465 \mathrm{~nm}$ as seen from Figure 1d. Their absorbance spectrum is starting at the point little blue-shifted from the band-edge PL peak of the 3 ML CdSe NPLs as expected (Figure 1d). The samples including undoped $\mathrm{CdSe} / \mathrm{CdS}$ NPLs dispersed in toluene have the PL peak at around $660 \mathrm{~nm}$, which is significantly red-shifted with respect to the PL peak of the doped samples. The PL spectra of the doped and undoped samples do not significantly overlap at the wavelength of peak intensity of donor Cu-doped NPLs (620 $\mathrm{nm})$. However, the small amount of overlap was further investigated by conducting the experiments at the second absorption peak of the undoped NPLs $(598 \mathrm{~nm})$. As shown in Figure 1d, absorbance spectrum of the acceptor undoped NPLs exhibits significant overlap with the emission of donor $\mathrm{Cu}$-doped NPLs, which is a favorable condition along with the absence of cross-talk in the PL spectra to achieve efficient NRET in these systems.

A systematic study of the NRET in the hybrid films of the doped and undoped NPLs was performed first by doing the steady-state PL measurements with the excitation pumping at $350 \mathrm{~nm}$. The PL spectra of only-donor NPLs, only-acceptor NPLs and the hybrid of these NPLs with the molar D/A ratio of $2.5 \times 10^{-5}$ were obtained as presented in Figure 2. PL emission

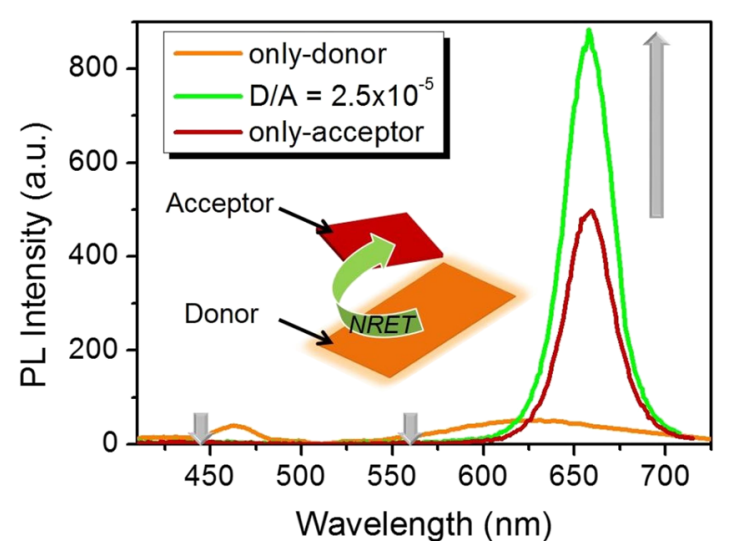

Figure 2. Steady-state PL spectra of the solid films of only-donor (orange), only-acceptor (red), and donor/acceptor having D/A $=2.5 \times$ $10^{-5}$ (green) cases.

of the donor NPLs originated from the band-edge and $\mathrm{Cu}$ related states is strongly quenched to almost zero level in the hybrid film, resulting in a significant enhancement in the acceptor PL emission which is arising from the acceptor bandedge. Because the D/A NPLs are in close proximity in their thin film ensembles, the energy transfer is expected to dominate by the NRET mechanism and negligibly affected by radiative energy transfer mechanism including photon emissionabsorption processes. The PL intensities of the only-donor and hybrid samples suggest the energy transfer efficiency as $\sim 93 \%$. This is a significantly high value for this kind of colloidal nanocrystal hybrid systems. In order to further analyze the potential of this new doped/undoped hybrid NPL samples and to reveal directly the NRET efficiencies and rates, we systematically studied the time-resolved PL kinetics in the samples with varying $\mathrm{D} / \mathrm{A}$ ratio along with the only-donor and only-acceptor cases.

We performed the TRF measurements by using a picosecond pulsed laser with an output photon energy of $3.307 \mathrm{eV}(375 \mathrm{~nm})$ integrated to a time-correlated single photon counting system (Experimental Section). The PL decay curves were obtained at room temperature and at low power intensity for various D/A molar ratios. The TRF measurements were carried out at the $\mathrm{Cu}$-state-related PL peak of the donor NPLs $(620 \mathrm{~nm})$ and at the second excitonic absorption peak of the acceptor NPLs (598 $\mathrm{nm})$. The PL decay curves at the band-edge peak emission wavelength of the donor NPLs $(465 \mathrm{~nm})$ were also measured (Supporting Information, Figure S1). Furthermore, decay 


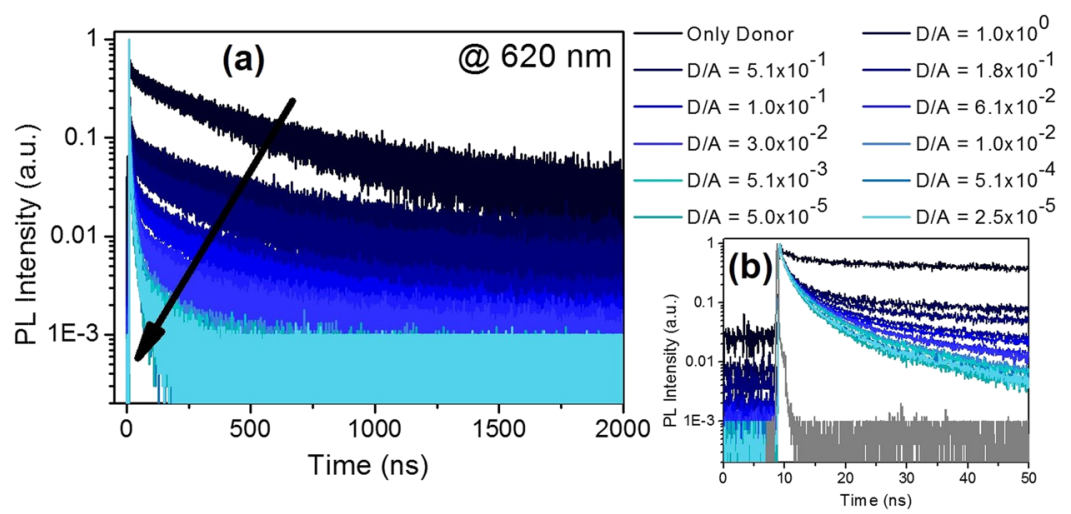

Figure 3. (a) Time-resolved PL decay curves at the donor trap emission peak wavelength, $620 \mathrm{~nm}$, for various D/A concentration ratios. (b) The zoom-in representation of the same decay curves together with the IRF having gray color.

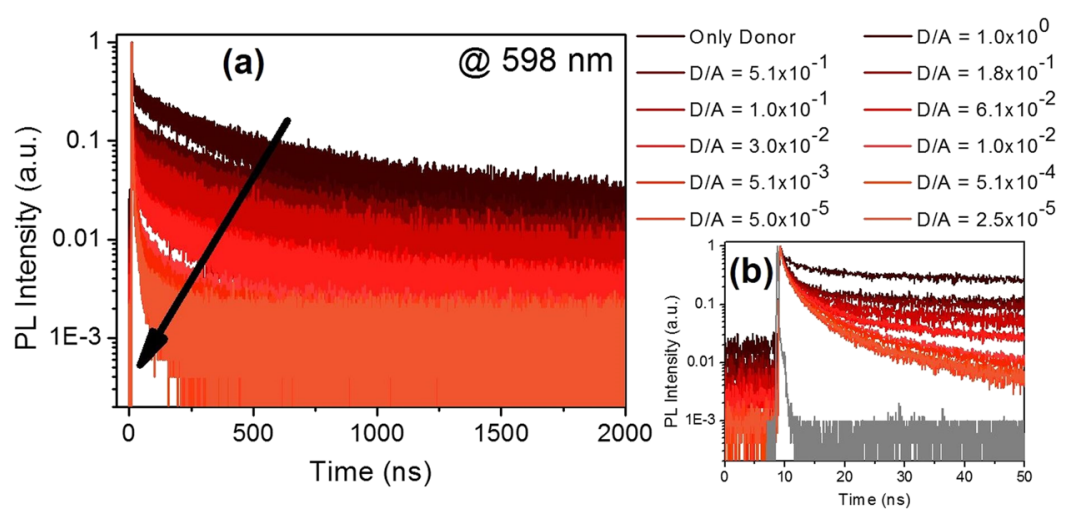

Figure 4. (a) Time-resolved PL decay curves at the acceptor second excitonic absorption peak wavelength ( $598 \mathrm{~nm}$ ) for various D/A ratios. (b) The zoom-in representation of the same decay curves together with the IRF having gray color.

curves for the band-edge emission of the acceptor at $660 \mathrm{~nm}$ were also recorded for the various $\mathrm{D} / \mathrm{A}$ cases; however, because of the cross-talk at the PL emissions of the donor and acceptor, not quantitative but the qualitative analysis of the decays at this wavelength is presented in Figure S2 in the Supporting Information.

Figure 3 shows the time-resolved PL decay curves of the samples having discrete D/A molar ratios at the peak PL wavelength of the donor, which is around $620 \mathrm{~nm}$. As depicted in Figure 3a, PL decay of the donor NPLs becomes faster as the acceptor concentration in the hybrid film increases. It is also seen from the first few nanoseconds of the curves presented in Figure $3 \mathrm{~b}$ along with the instrument response function (IRF) curve that this behavior is obviously not in the limits of our experimental system. This feature of the curves is a strong evidence of opening a new nonradiative channel for the excited state energy relaxation in the donor NPLs due to the acceptor NPLs around in close proximity.

In order to further examine the findings from the PL decay curves at the donor $\mathrm{Cu}$-related peak emission wavelength (620 $\mathrm{nm}$ ), we performed the time-resolved PL spectroscopy with the same samples having discrete $\mathrm{D} / \mathrm{A}$ ratios but at a different emission wavelength, which is the wavelength of the acceptor second absorption peak (598 nm). Besides the almost zero cross-talk of the donor and acceptor emissions, another reason for choosing this wavelength is to analyze the possible effect of the very small overlapping in the donor emission with the acceptor emission at $620 \mathrm{~nm}$. As shown in Figure 4a, faster decays were observed at $598 \mathrm{~nm}$ with the decreasing D/A molar ratio, which is an agreed result previously obtained at $620 \mathrm{~nm}$ (see Figure 3). The curves are also far from the limits of the timeresolved PL instrumentation as shown in Figure $4 \mathrm{~b}$.

The time-resolved PL decay curves obtained at the wavelengths of 620 and $598 \mathrm{~nm}$ were analyzed by fitting with a multiexponential decay function including four exponential components. Four different components in the fitting function can be attributed to four completely independent or partially dependent decay channels in the NPL and it is an intrinsic property of the colloidal nanomaterials as it was previously observed in the literature. ${ }^{9,36}$ The amplitude-averaged PL lifetimes $\left(\tau_{\text {av }}\right)$ along with the fitting parameters for both the wavelengths of the donor NPLs are presented in Tables S1 and S2 (Supporting Information). The PL intensity of only-donor solid film exhibits an average decay lifetime of $191.9 \mathrm{~ns}$ at 620 $\mathrm{nm}$ and $127.4 \mathrm{~ns}$ at $598 \mathrm{~nm}$. These average decay lifetimes decrease to $2.2 \mathrm{~ns}$ at $620 \mathrm{~nm}$ and $2.1 \mathrm{~ns}$ at $598 \mathrm{~nm}$ in the sample with $\mathrm{D} / \mathrm{A}=5.0 \times 10^{-5}$, in which there is a large number of acceptors per donor, and they saturate at around these values. Major modifications in the PL lifetimes of the donor as a function of the increasing acceptor-to-donor ratio are strong indications of the NRET from the donor (e.g. Cu-doped NPLs) to the acceptor (e.g. undoped core/shell NPLs). Further analysis of the donor PL lifetimes provides the NRET rates and efficiencies, which are presented in Table 1 . The analyses of the NRET rates were computed by using the eq 1

$$
\gamma_{\mathrm{NRET}}=\frac{1}{\tau_{\mathrm{DA}}}-\frac{1}{\tau_{\mathrm{D}}}
$$


Table 1. PL Decay Lifetimes $(\tau)$, NRET Rates $\left(\gamma_{\text {NRET }}\right)$ and NRET Efficiencies $\left(\eta_{\text {NRET }}\right)$ of the Donor NPLs for the Samples with Varying D/A Ratios

\begin{tabular}{ccccccc}
$\tau_{\text {av donor }}$ & $\begin{array}{c}\tau_{\text {av donor }} \\
(\mathrm{ns})\end{array}$ & $\begin{array}{c}\gamma_{\text {NRET }} \\
\left(\mathrm{ns}^{-1}\right)\end{array}$ & $\begin{array}{c}\gamma_{\text {NRET }} \\
\left(\mathrm{ns}^{-1}\right)\end{array}$ & $\begin{array}{c}\eta_{\text {NRET }}(\%) \\
598\end{array}$ & $\begin{array}{c}\eta_{\text {NRET }} \\
(\%)\end{array}$ \\
$\begin{array}{c}\text { D/Aratio } \\
2.5 \times 10^{-5}\end{array}$ & $620 \mathrm{~nm}$ & $598 \mathrm{~nm}$ & $620 \mathrm{~nm}$ & $598 \mathrm{~nm}$ \\
$5.0 \times 10^{-5}$ & 2.4 & 2.3 & 0.419 & 0.427 & 99 & 98 \\
$5.1 \times 10^{-4}$ & 2.5 & 2.1 & 0.456 & 0.468 & 99 & 98 \\
$5.1 \times 10^{-3}$ & 2.9 & 3.5 & 0.401 & 0.394 & 99 & 98 \\
$1.0 \times 10^{-2}$ & 3.1 & 4.1 & 0.341 & 0.262 & 99 & 97 \\
$3.0 \times 10^{-2}$ & 4.5 & 9.4 & 0.219 & 0.099 & 98 & 93 \\
$6.1 \times 10^{-2}$ & 5.1 & 10.1 & 0.191 & 0.091 & 97 & 92 \\
$1.0 \times 10^{-1}$ & 9 & 15.7 & 0.106 & 0.056 & 95 & 88 \\
$1.8 \times 10^{-1}$ & 10 & 22.5 & 0.095 & 0.037 & 95 & 82 \\
$5.1 \times 10^{-1}$ & 21 & 37.7 & 0.042 & 0.019 & 89 & 70 \\
$1.1 \times 10^{0}$ & 34.2 & 44.9 & 0.024 & 0.014 & 82 & 65 \\
only donor & 191.9 & 127.4 & & & & \\
\hline
\end{tabular}

where $\tau_{\mathrm{DA}}$ and $\tau_{\mathrm{D}}$ are the amplitude-averaged PL lifetimes of the donor NPLs in the presence and absence of acceptor NPLs, respectively. As seen from the Table 1, the NRET rates at both the wavelengths, 620 and $598 \mathrm{~nm}$, increase with the number of acceptor per donor, reaching to the maximum value of around 0.46 . The record NRET efficiencies were also calculated by using the following relation

$$
\eta_{\mathrm{NRET}}=\frac{\gamma_{\mathrm{NRET}}}{\gamma_{\mathrm{NRET}}+\tau_{\mathrm{D}}^{-1}}=1-\frac{\tau_{\mathrm{DA}}}{\tau_{\mathrm{D}}}
$$

Figure 5 depicts the NRET efficiencies as a function of the varying ratio of $\mathrm{D} / \mathrm{A}$ molar concentration. Progressive increase

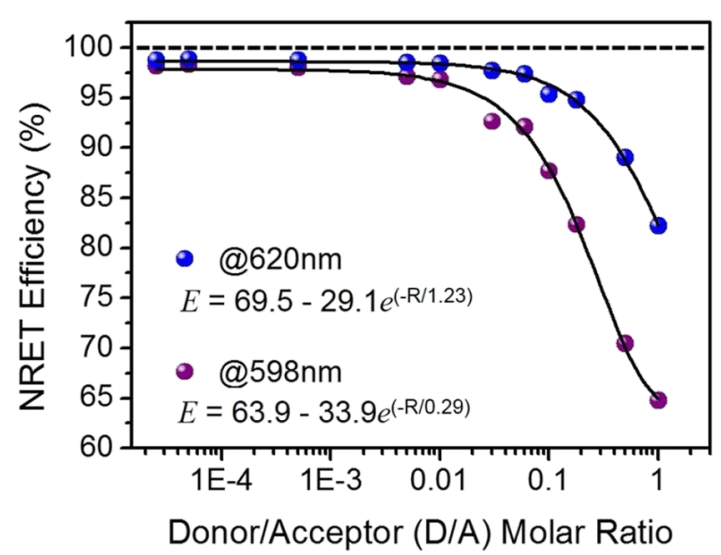

Figure 5. NRET efficiency as a function of D/A molar ratio.

saturating at high efficiency levels with the decreasing D/A ratio is observed because of the large absorption cross-section of the acceptor NPLs. As seen from Figure 5, the saturations occur at around $99 \%$ (for the emission wavelength of $620 \mathrm{~nm}$ ) and at around $98 \%$ (for the emission wavelength of $598 \mathrm{~nm}$ ). As for another perspective discussed in the literature, besides these results from the average lifetimes, NRET efficiency for the sample having the $\mathrm{D} / \mathrm{A}$ molar ratio of $2.5 \times 10^{-5}$ was also obtained as around $93 \%$ by using the second lifetime components, $\tau_{2}$, at both the wavelengths of 620 and $598 \mathrm{~nm}$. Among all the lifetime components, the second component was used for this analysis because of its strong attribution to the $\mathrm{Cu}$ related emission in the literature. ${ }^{26,29}$ These are, to the best of our knowledge, the highest reported NRET efficiencies in the colloidal doped-undoped quasi-2D quantum well semiconductor nanocrystal hybrids (e.g. doped NPL-undoped NPL). Previously, the NRET efficiency was reported for the undoped QD-QD donor/acceptor pairs in excess of $90 \% ;^{37,38}$ for the undoped QD-NPL pairs up to $90 \%$; $^{39}$ and for the undoped NPL-NPL pairs up to $60^{12}$ and $90 \%$ (for a single NPL-NPL pair $)^{4}$ at room temperature. Another important point is the saturation molar ratio value of the efficiencies, which is beginning at $\sim 0.1$ and reaching to the saturation value at $\sim 0.02$. From the surface calculations, we also obtained the saturation value at $\sim 0.02$, which is in good agreement with the experimental results in Figure 5.

Further analysis was performed on the NRET efficiencies including single donor and multiple numbers of acceptor to obtain the efficiency for a single donor-acceptor (D-A) pair in the ensemble of NPLs. To this end, we used a well-known model developed by Raicu. ${ }^{40,41}$ As presented in Figure 6, efficiency per

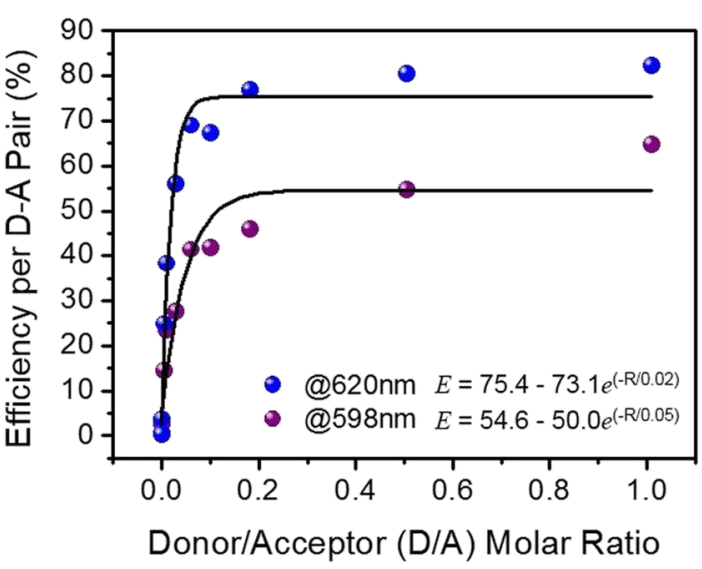

Figure 6. NRET efficiency for a single D/A pair as a function of D/A molar ratio.

$\mathrm{D}-\mathrm{A}$ pair was calculated which is almost the same up to a saturation value of the $\mathrm{D} / \mathrm{A}$ molar ratio and then it rapidly decreases to very small values of around zero. The decreasing behavior in the pair efficiencies is due to the approximation of constant dependence of the NRET to the distance between donor and acceptor in a pair. This drawback of the model is favorable for us to observe the $\mathrm{D} / \mathrm{A}$ ratio of a single pair at the efficiency saturation point, which is significant to explain the behavior in the NRET efficiencies from the ensemble measurements (Figure 5). The saturation values from the ensemble measurements and the single-pair calculations $(\sim 0.1)$ are consistent with each other. This method provided useful and important evaluation of the NRET efficiencies from our ensemble measurements.

For further investigation of the NRET, PL excitation (PLE) spectroscopy was carried out in the hybrid solid films along with the only-donor and only-acceptor samples. The PLE spectra of the bare donor and acceptor films, which were measured at their peak PL wavelengths, are shown in Figure 7a. As seen from the figure, the excitation spectra exhibit similar features as the absorption spectra of the NPLs (see Figure 1d). After this confirmation of the PLE spectra, we measured the excitation spectra of the hybrid films having discrete D/A molar ratios: 1.0 $\times 10^{0}, 1.0 \times 10^{-1}, 1.0 \times 10^{-2}$, and $5.1 \times 10^{-4}$. Figure $7 \mathrm{~b}$ depicts the excitation spectra of the hybrid NPL films, which are measured at the acceptor peak emission wavelength, $660 \mathrm{~nm}$. These PLE spectra are normalized at the second excitonic 
(a)

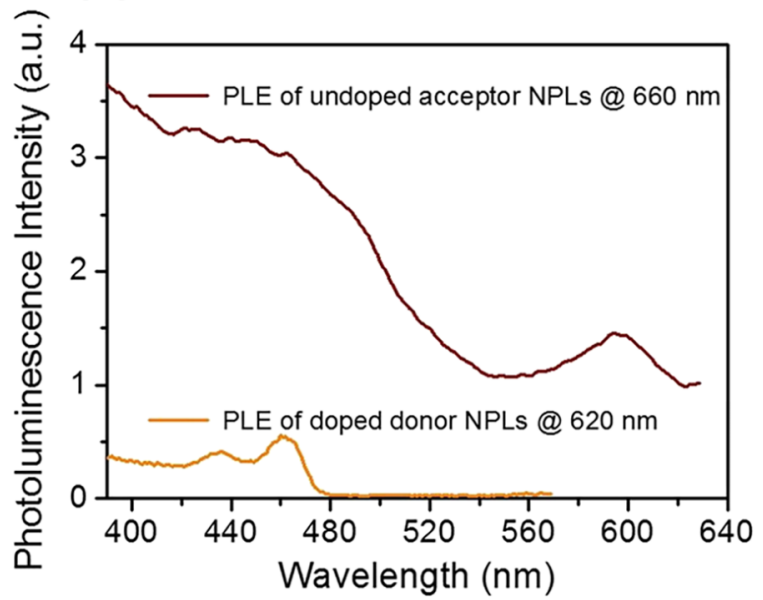

(b)

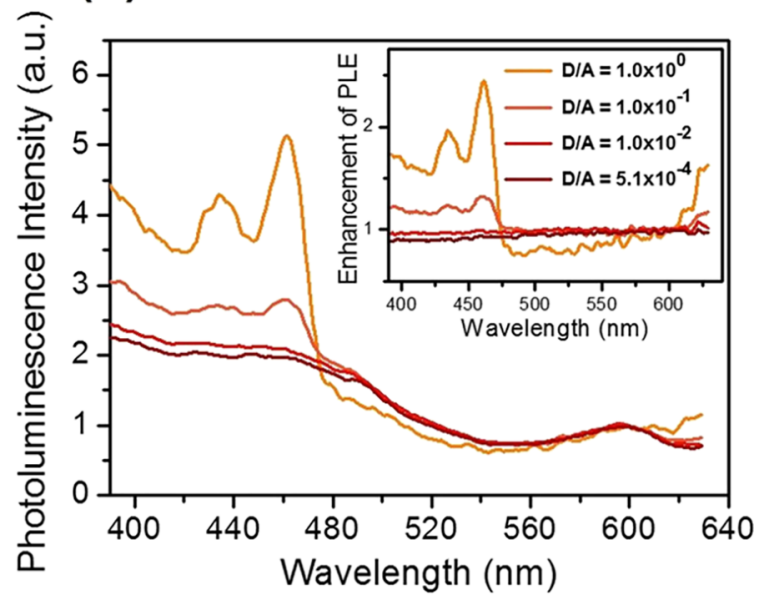

Figure 7. (a) PLE spectra of the doped donor NPLs (orange) and the undoped acceptor NPLs (red) measured at their Cu-related emission peak wavelength $(620 \mathrm{~nm})$ and excitonic emission peak wavelength $(660 \mathrm{~nm})$, respectively. (b) PLE spectra of the samples having different D/A ratios, measured at $660 \mathrm{~nm}$ and then normalized at the second excitonic (e-lh transition) peak wavelength ( $598 \mathrm{~nm})$ of the acceptor. Inset figure shows the enhancement of PLE through the NRET in the hybrid donor/acceptor samples.

absorption peak $(\sim 598 \mathrm{~nm})$ of the acceptor NPLs because the donor NPLs do not possess absorption at this spectral region (see Figure 1d). These normalized excitation spectra of the NPLs show gradual enhancement in the PL intensity at shorter wavelengths with increasing number of donor NPLs per an acceptor NPL. This result is in good agreement with the phenomenon of exciton funneling from the donor to the acceptor. The PLE spectra were further examined for the enhancements in the hybrid samples by dividing the normalized excitation spectra of the hybrids with those of the only-acceptor sample. The relative variations in the acceptor emission through the NRET are represented for the corresponding molar ratios in the inset of Figure $7 \mathrm{~b}$. With the increasing number of donordoped NPLs relative to the acceptor undoped NPLs; the PLE spectrum resembles the absorption features of the donor NPLs.

\section{CONCLUSIONS}

We studied the efficiency of excitonic energy transfer between colloidal $\mathrm{Cu}$-doped core $\mathrm{CdSe}$ and undoped core/shell CdSe/ CdS NPLs. Using steady-state and time-resolved PL spectroscopy techniques, we showed the PL quenching of the donor $\mathrm{Cu}$ doped NPLs and the systematic modifications in the timeresolved PL kinetics when the acceptor undoped NPLs are located in close proximity. The record level energy transfer efficiency in this newly shown all-colloidal and all-quasi-2D doped/undoped hybrid system was presented to be around 99\% at room temperature. All these findings provide fundamentally important knowledge for the NRET dynamics of quasi-2D colloidal hybrid material systems. We also believe that such strongly efficient NRET in these quantum-confined structures will enable high-performance photonics and optoelectronics including Cu-doped NPLs.

\section{ASSOCIATED CONTENT}

\section{S Supporting Information}

The Supporting Information is available free of charge on the ACS Publications website at DOI: 10.1021/acs.jpcc.8b10177.

Time-resolved PL decay curves at the donor band-edge emission peak wavelength $(465 \mathrm{~nm})$ for various $\mathrm{D} / \mathrm{A}$ ratios; time-resolved PL decay curves at the acceptor band-edge emission peak wavelength $(660 \mathrm{~nm})$ for various D/A ratios; fitting parameters and the amplitude-averaged PL lifetimes $\left(\tau_{\mathrm{av}}\right)$ of the donor colloidal CdSe 3ML doped NPLs at the detection wavelength of $620 \mathrm{~nm}$; and fitting parameters and the amplitudeaveraged PL lifetimes $\left(\tau_{\mathrm{av}}\right)$ of the donor colloidal CdSe 3 ML doped NPLs at the detection wavelength of $598 \mathrm{~nm}$ (PDF)

\section{AUTHOR INFORMATION}

\section{Corresponding Author}

*E-mail: volkan@stanfordalumni.org, hvdemir@ntu.edu.sg. Phone: +90 312-290-1021.

\section{ORCID}

Murat Olutas: 0000-0002-6250-6977

Hilmi Volkan Demir: 0000-0003-1793-112X

\section{Author Contributions}

The manuscript was written through contributions of all authors. All authors have given approval to the final version of the manuscript.

Notes

The authors declare no competing financial interest.

\section{ACKNOWLEDGMENTS}

The authors would like to thank for the financial support from TÜBİTAK 115E679. H.V.D. also gratefully acknowledges support from TUBA.

\section{REFERENCES}

(1) Ithurria, S.; Dubertret, B. Quasi 2D Colloidal CdSe Platelets with Thicknesses Controlled at the Atomic Level. J. Am. Chem. Soc. 2008, 130, 16504-16505.

(2) Ithurria, S.; Tessier, M. D.; Mahler, B.; Lobo, R. P. S. M.; Dubertret, B.; Efros, A. L. Colloidal Nanoplatelets with TwoDimensional Electronic Structure. Nat. Mater. 2011, 10, 936-941.

(3) Kunneman, L. T.; Schins, J. M.; Pedetti, S.; Heuclin, H.; Grozema, F. C.; Houtepen, A. J.; Dubertret, B.; Siebbeles, L. D. A. Nature and Decay Pathways of Photoexcited States in CdSe and CdSe/CdS Nanoplatelets. Nano Lett. 2014, 14, 7039-7045. 
(4) Rowland, C. E.; Fedin, I.; Zhang, H.; Gray, S. K.; Govorov, A. O.; Talapin, D. V.; Schaller, R. D. Picosecond Energy Transfer and Multiexciton Transfer Outpaces Auger Recombination in Binary CdSe Nanoplatelet Solids. Nat. Mater. 2015, 14, 484-489.

(5) Chen, Z.; Nadal, B.; Mahler, B.; Aubin, H.; Dubertret, B. Quasi-2D Colloidal Semiconductor Nanoplatelets for Narrow Electroluminescence. Adv. Funct. Mater. 2013, 24, 295-302.

(6) Rajadell, F.; Climente, J. I.; Planelles, J. Excitons in Core-Only, Core-Shell and Core-Crown CdSe Nanoplatelets: Interplay between in-Plane Electron-Hole Correlation, Spatial Confinement, and Dielectric Confinement. Phys. Rev. B 2017, 96, 035307.

(7) Tessier, M. D.; Mahler, B.; Nadal, B.; Heuclin, H.; Pedetti, S.; Dubertret, B. Spectroscopy of Colloidal Semiconductor Core/Shell Nanoplatelets with High Quantum Yield. Nano Lett. 2013, 13, 33213328.

(8) Richter, M. Nanoplatelets as Material System between Strong Confinement and Weak Confinement. Phys. Rev. Mater. 2017, 1, 016001.

(9) Tessier, M. D.; Javaux, C.; Maksimovic, I.; Loriette, V.; Dubertret, B. Spectroscopy of Single CdSe Nanoplatelets. ACS Nano 2012, 6, 6751-6758.

(10) Olutas, M.; Guzelturk, B.; Kelestemur, Y.; Yeltik, A.; Delikanli, S.; Demir, H. V. Lateral Size-Dependent Spontaneous and Stimulated Emission Properties in Colloidal CdSe Nanoplatelets. ACS Nano 2015, 9, 5041-5050.

(11) Yeltik, A.; Delikanli, S.; Olutas, M.; Kelestemur, Y.; Guzelturk, B.; Demir, H. V. Experimental Determination of the Absorption CrossSection and Molar Extinction Coefficient of Colloidal CdSe Nanoplatelets. J. Phys. Chem. C 2015, 119, 26768-26775.

(12) Guzelturk, B.; Olutas, M.; Delikanli, S.; Kelestemur, Y.; Erdem, O.; Demir, H. V. Nonradiative Energy Transfer in Colloidal CdSe Nanoplatelet Films. Nanoscale 2015, 7, 2545-2551.

(13) Schmid, G. Nanoparticles: From Theory to Application, 2nd ed.; Wiley-VCH Verlag GmbH \& Co. KGaA, 2010.

(14) Nanocrystal Quantum Dots; Klimov, V. I., Ed.; CRC Press, 2010.

(15) Kamat, P. V. Quantum Dot Solar Cells. Semiconductor Nanocrystals as Light Harvesters. J. Phys. Chem. C 2008, 112, $18737-18753$.

(16) Horoz, S.; Dai, Q.; Maloney, F. S.; Yakami, B.; Pikal, J. M.; Zhang, X.; Wang, J.; Wang, W.; Tang, J. Absorption Induced by Mn Doping of $\mathrm{ZnS}$ for Improved Sensitized Quantum-Dot Solar Cells. Phys. Rev. Appl. 2015, 3, 024011.

(17) Bradshaw, L. R.; Knowles, K. E.; McDowall, S.; Gamelin, D. R. Nanocrystals for Luminescent Solar Concentrators. Nano Lett. 2015, $15,1315-1323$.

(18) Zhang, W.; Lou, Q.; Ji, W.; Zhao, J.; Zhong, X. Color-Tunable Highly Bright Photoluminescence of Cadmium-Free Cu-Doped Zn-InS Nanocrystals and Electroluminescence. Chem. Mater. 2013, 26, 1204-1212.

(19) Xu, S.; Wang, C.; Wang, Z.; Zhang, H.; Yang, J.; Xu, Q.; Shao, H.; Li, R.; Lei, W.; Cui, Y. Aqueous Synthesis of Internally Doped $\mathrm{Cu}: \mathrm{ZnSe} / \mathrm{ZnS}$ Core-Shell Nanocrystals with Good Stabilit0079. Nanotechnology 2011, 22, 275605.

(20) Erickson, C. S.; Bradshaw, L. R.; McDowall, S.; Gilbertson, J. D.; Gamelin, D. R.; Patrick, D. L. Zero-Reabsorption Doped-Nanocrystal Luminescent Solar Concentrators. ACS Nano 2014, 8, 3461-3467.

(21) Morgan, D. P.; Maddux, C. J. A.; Kelley, D. F. Transient Absorption Spectroscopy of CdSe Nanoplatelets. J. Phys. Chem. C 2018, 122, 23772-23779.

(22) Guzelturk, B.; Erdem, O.; Olutas, M.; Kelestemur, Y.; Demir, H. V. Stacking in Colloidal Nanoplatelets: Tuning Excitonic Properties. ACS Nano 2014, 8, 12524-12533.

(23) Grim, J. Q.; Christodoulou, S.; Di Stasio, F.; Krahne, R.; Cingolani, R.; Manna, L.; Moreels, I. Continuous-Wave Biexciton Lasing at Room Temperature Using Solution-Processed Quantum Wells. Nat. Nanotechnol. 2014, 9, 891-895.

(24) She, C.; Fedin, I.; Dolzhnikov, D. S.; Demortière, A.; Schaller, R. D.; Pelton, M.; Talapin, D. V. Low-Threshold Stimulated Emission Using Colloidal Quantum Wells. Nano Lett. 2014, 14, 2772-2777.
(25) Zhang, F.; Wang, S.; Wang, L.; Lin, Q.; Shen, H.; Cao, W.; Yang, C.; Wang, H.; Yu, L.; Du, Z.; et al. Super Color Purity Green Quantum Dot Light-Emitting Diodes Fabricated by Using CdSe/CdS Nanoplatelets. Nanoscale 2016, 8, 12182-12188.

(26) Sharma, M.; Gungor, K.; Yeltik, A.; Olutas, M.; Guzelturk, B.; Kelestemur, Y.; Erdem, T.; Delikanli, S.; McBride, J. R.; Demir, H. V. Near-Unity Emitting Copper-Doped Colloidal Semiconductor Quantum Wells for Luminescent Solar Concentrators. Adv. Mater. 2017, 29, 1700821.

(27) Sharma, M.; Olutas, M.; Yeltik, A.; Kelestemur, Y.; Sharma, A.; Delikanli, S.; Guzelturk, B.; Gungor, K.; McBride, J. R.; Demir, H. V. Understanding the Journey of Dopant Copper Ions in Atomically Flat Colloidal Nanocrystals of CdSe Nanoplatelets Using Partial Cation Exchange Reactions. Chem. Mater. 2018, 30, 3265-3275.

(28) Knowles, K. E.; Nelson, H. D.; Kilburn, T. B.; Gamelin, D. R. Singlet-Triplet Splittings in the Luminescent Excited States of Colloidal $\mathrm{Cu}+$ : CdSe, $\mathrm{Cu}+$ :InP, and CuInS 2 Nanocrystals: ChargeTransfer Configurations and Self-Trapped Excitons. J. Am. Chem. Soc. 2015, 137, 13138-13147.

(29) Knowles, K. E.; Hartstein, K. H.; Kilburn, T. B.; Marchioro, A.; Nelson, H. D.; Whitham, P. J.; Gamelin, D. R. Luminescent Colloidal Semiconductor Nanocrystals Containing Copper: Synthesis, Photophysics, and Applications. Chem. Rev. 2016, 116, 10820-10851.

(30) Valeur, B. Molecular Fluorescence: Principles and Applications; WILEY-VCH, 2002.

(31) Medintz, I.; Hildebrandt, N. FRET-Förster Resonance Energy Transfer; Wiley-VCH Verlag GmbH \& Co. KGaA, 2014.

(32) Govorov, A.; Martínez, P. L. H.; Demir, H. V. Understanding and Modeling FRETFörster-Type Resonance Energy Transfer: Introduction to Forster-Type Resonance Energy Transfer (FRET) FRET Series; Springer, 2016.

(33) Sarkar, S.; Maity, A. R.; Karan, N. S.; Pradhan, N. Fluorescence Energy Transfer from Doped to Undoped Quantum Dots. J. Phys. Chem. C 2013, 117, 21988-21994.

(34) Chen, D.; Gao, Y.; Chen, Y.; Ren, Y.; Peng, X. Structure Identification of Two-Dimensional Colloidal Semiconductor Nanocrystals with Atomic Flat Basal Planes. Nano Lett. 2015, 15, 44774482.

(35) Ithurria, S.; Talapin, D. V. Colloidal Atomic Layer Deposition (cALD) Using Self-Limiting Reactions at Nanocrystal Surface Coupled to Phase Transfer between Polar and Nonpolar Media. J. Am. Chem. Soc. 2012, 134, 18585-18590.

(36) Durisic, N.; Godin, A. G.; Walters, D.; Grütter, P.; Wiseman, P. W.; Heyes, C. D. Probing the "Dark" Fraction of Core-Shell Quantum Dots by Ensemble and Single Particle pH-Dependent Spectroscopy. ACS Nano 2011, 5, 9062-9073.

(37) Achermann, M.; Petruska, M. A.; Crooker, S. A.; Klimov, V. I. Picosecond Energy Transfer in Quantum Dot Langmuir-Blodgett Nanoassemblies. J. Phys. Chem. B 2003, 107, 13782-13787.

(38) De Benedetti, W. J. I.; Nimmo, M. T.; Rupich, S. M.; Caillard, L. M.; Gartstein, Y. N.; Chabal, Y. J.; Malko, A. V. Efficient Directed Energy Transfer through Size-Gradient Nanocrystal Layers into Silicon Substrates. Adv. Funct. Mater. 2014, 24, 5002-5010.

(39) Olutas, M.; Guzelturk, B.; Kelestemur, Y.; Gungor, K.; Demir, H. V. Highly Efficient Nonradiative Energy Transfer from Colloidal Semiconductor Quantum Dots to Wells for Sensitive Noncontact Temperature Probing. Adv. Funct. Mater. 2016, 26, 2891-2899.

(40) Clapp, A. R.; Medintz, I. L.; Mauro, J. M.; Fisher, B. R.; Bawendi, M. G.; Mattoussi, H. Fluorescence Resonance Energy Transfer Between Quantum Dot Donors and Dye-Labeled Protein Acceptors. J. Am. Chem. Soc. 2004, 126, 301-310.

(41) Raicu, V. Efficiency of Resonance Energy Transfer in HomoOligomeric Complexes of Proteins. J. Biol. Phys. 2007, 33, 109-127. 